\title{
Blendas de Poliamida 6/Elastômero: Propriedades e Influência da Adição de Agente Compatibilizante
}

\author{
Guilherme M. O. Barra, Jerusa Roeder, Valdir Soldi, Alfredo T. N. Pires \\ Grupo de Estudo em Materiais Poliméricos, UFSC \\ José A. M. Agnelli \\ Departamento de Engenharia de Materiais, UFSCar
}

\begin{abstract}
Resumo: Neste trabalho foram estudadas as propriedades micro e macroscópicas de misturas físicas binárias de poliamida 6 [PA6] com copolímero poli(etileno-co-propileno-co-dieno) [EPDM] e em presença de pequenas quantidades de EPDM ou EPM enxertados com anidrido maleico, atuando como agentes compatibilizantes. Os componentes puros (poliamida 6 e EPDM) e as blendas poliméricas em diferentes composições foram analisados por calorimetria diferencial de varredura (DSC) e espectroscopia de infravermelho (FTIR). Os resultados indicaram a imiscibilidade dos componentes da mistura em toda a faixa de composição estudada. A utilização das técnicas de análise elementar, microscopia eletrônica de varredura e propriedades mecânicas permitiu avaliar a homogeneidade da mistura, a redução do tamanho dos domínios do elastômero e o aumento da força de impacto da matriz de PA6 com a adição do agente compatibilizante à mistura binária de PA6/EPDM.
\end{abstract}

Palavras-chave: Poliamida 6, EPDM, anidrido maleico, blendas poliméricas.

\section{Polyamide 6/Elastomer blends: Properties and compatibilizer influence}

Abstract: This study describes the micro- and macroscopic properties of polyamide 6 (PA6) and ethylene /propylene/ diene copolymer (EPDM) blends, and the effects from small quantities of EPDM or EPM grafted with maleic anhydride in this polymer mixture. The components and polymer blends at different compositions were analyzed by differential scanning calorimeter (DSC) and infrared spectroscopy (FTIR). The results suggest immiscible mixture in all ranges of the blend's compositions. Scanning electron microscopy (SEM) micrographs of the blends indicated that the size of rubber aggregates decreases as the functionalized elastomer (EPDM-g-MA or EPM-g-MA) is added to the PA6/EPDM mixture, and the impact strength of the PA6 matrix increases significantly.

Keywords: Polyamide 6, EPDM, maleic anhydride, blends.

\section{Introdução}

Desde o início do século passado o desenvolvimento de materiais poliméricos tem se expandido bastante, o que pode ser observado no nosso dia a dia, através da composição dos inúmeros utilitários que nos cercam. Os processos de polimerização foram desenvolvidos e uma grande quantidade de novos polímeros surgiram. Em algumas situações, ao invés de desenvolver uma rota sintética para a obtenção de um novo material polimérico, pesquisa e desenvolvimento são direcionados ao estudo de misturas físicas de dois ou mais polímeros, ou seja, blendas poliméricas. Entretanto, a aplicação de alguns polímeros fica limitada pelas características e desempenho sob impacto nas condições de temperatura ambiente. Esta situação se agrava, principalmente para temperaturas de utilização abaixo de zero graus Celsius ${ }^{[1]}$. Uma maneira de contornar esta limitação é preparar blendas poliméricas, em que um dos compo- nentes é um elastômero. Dependendo dos componentes poliméricos, a mistura pode se apresentar de forma não homogênea, com domínios grandes, sendo então classificada como uma blenda imiscível. Blendas com esta característica apresentam alta tensão interfacial e fraca adesão entre a matriz e a fase dispersa. Estudos têm sido direcionados para avaliar a forma, tamanho e distribuição dos domínios da fase dispersa, relacionando-os com as propriedades macroscópicas do material resultante. A adição de pequenas quantidades de um terceiro componente à blenda imiscível, pode alterar a energia interfacial e a dispersão entre as fases, atuando como um agente compatibilizante. Os agentes compatibilizantes geralmente são copolímeros em bloco ou enxertados e podem provocar um decréscimo no tamanho da fase dispersa e modificar a tenacidade do material em relação ao termoplástico ${ }^{[2]}$. Por exemplo, o copolímero em bloco poli(náilon6-co-étersulfona-co-náilon6) tem sido utilizado como agente compatibilizante para

Autor para correspondência: Alfredo T. N. Pires, Departamento de Química, UFSC, Campus Universitário Trindade, CEP: 88040-900, Florianópolis, SC. E-mail:pires@qmc.ufsc.br 
a blenda binária poli(étersulfona)/náilon6 ${ }^{[3]}$ e EPDM enxertado com anidrido maleico em blendas de $\mathrm{PA} 6 / \mathrm{PC}^{[4]} \mathrm{e}$ $\mathrm{PA}-6 / \mathrm{EPM}^{[5,6]}$.

Trabalhos anteriores de nosso grupo de pesquisa têm mostrado a influência da adição de polipropileno enxertado com anidrido maleico [PP-g-MA] às blendas de PP/PA6 ou PP/PU ${ }^{[7,8]}$. Nestes dois sistemas foi observada a formação de um copolímero na interface entre os domínios e a matriz através do aparecimento da ligação imídica. Pagnoulle e Jérôme também observaram a reação entre o anidrido maleico e a amina primária do copolímero nas blendas de poli(etileno-copropileno-co-dieno)/poli(estireno-co-acrilonitrila) em presença de EPDM enxertado com anidrido maleico ${ }^{[9]}$.

Nos últimos anos tem sido estudada a utilização de elastômeros como modificadores de impacto de poliamidas para aplicações específicas ${ }^{[10,11]}$. Por exemplo, a presença de aditivos políméricos quimicamente reativos com PA6 e fisicamente compatíveis com a fase do poli(estireno-co-acrilonitrila), modificaram a tenacidade do material resultante ${ }^{[12]}$.

Este trabalho tem por objetivo avaliar o efeito da adição de EPDM ou EPM enxertado com anidrido maleico nas blendas binárias imiscíveis de PA6/EPDM sobre as propriedades micro e macroscópicas.

\section{Experimental}

\section{Materiais}

O copolímero EPDM utilizado neste trabalho apresenta temperatura de transição vítrea $\left(\mathrm{T}_{\mathrm{g}}\right)$ igual a $-50^{\circ} \mathrm{C}$ e foi gentilmente cedido pela Nitriflex, com uma percentagem de 1,7\% de etilideno norborneno segundo o fabricante. A estrutura do EPDM é apresentada a seguir, onde $\mathrm{m}, \mathrm{n}$ e $\mathrm{p}$ denotam os diferentes graus de polimerização.

Os copolímeros de EPDM enxertados com $0,4 \%$ e EPM com $0,7 \%$ de anidrido maleico foram gentilmente cedidos pela Uniroyal Chemical e EXXON Chemical, respectivamente. As amostras de EPDM-g-AM com 1,7\% de anidrido maleico foram obtidas conforme descrito na referência 13 . Poliamida 6 [PA6] com $\mathrm{T}_{\mathrm{g}}$ igual a $42{ }^{\circ} \mathrm{C}, \mathrm{T}_{\mathrm{c}}$ igual a $68{ }^{\circ} \mathrm{C}$ e $\mathrm{T}_{\mathrm{f}}$ igual a $225^{\circ} \mathrm{C}$ foi doada pela Petronyl Ind. Com. Todos os polímeros foram utilizados sem purificação adicional.

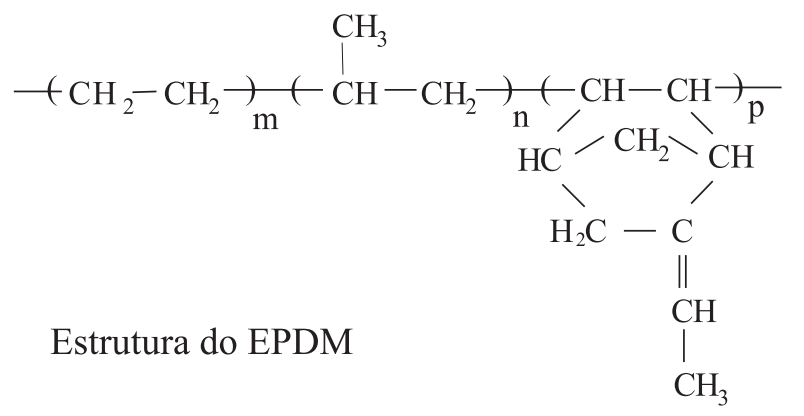

\section{Métodos}

Os polímeros foram previamente mantidos por $12 \mathrm{~h}$ a $100{ }^{\circ} \mathrm{C}$ sob vácuo e em seguida misturados no estado fun- dido em diferentes composições, numa extrusora CSI Max Extruder, modelo CS-194 A. As especificações experimentais da extrusora são: i) relação comprimento/diâmetro igual a 4; ii) velocidade de rotação de rosca de $70 \mathrm{rev} / \mathrm{min}$ e iii) duas zonas de aquecimento em $230^{\circ} \mathrm{C}$. O processo de extrusão foi repetido três vezes para se obter melhor homogeneidade entre as fases na mistura, que visualmente não indicou a ocorrência de decomposição. Foram preparadas blendas binárias de PA6/EPDM e PA6/EPM em presença do agente compatibilizante (elastômero enxertado com anidrido maleico), sendo as composições expressas em porcentagem em peso.

Os espectros de infravermelho dos polímeros puros e das blendas foram obtidos diretamente da superfície dos filmes utilizando acessórios de reflectância múltipla, em um espectrômetro de infravermelho 16 PC Perkin Elmer, com resolução de $4 \mathrm{~cm}^{-1}$, sendo realizadas 20 varreduras para cada espectro. Nas faixas de número de onda de interesse os espectros de infravermelho das blendas e dos componentes puros foram analisados a partir da deconvolução gaussiana, utilizando o programa Origin 4.1.

As curvas de análise térmica foram obtidas em um calorímetro Shimadzu 50, calibrado com índio e utilizando nitrogênio como gás de arraste. Os polímeros puros e blendas foram aquecidos a uma taxa de $10{ }^{\circ} \mathrm{C} / \mathrm{min}$ da temperatura ambiente até $250^{\circ} \mathrm{C}$ e mantidos nesta temperatura por 10 minutos, seguidos de um choque térmico em nitrogênio líquido. As amostras foram novamente aquecidas a uma taxa de $10^{\circ} \mathrm{C} / \mathrm{min}$ de $-80^{\circ} \mathrm{C}$ ou da temperatura ambiente até $250^{\circ} \mathrm{C}$, para os elastômeros e blendas, respectivamente.

Pequenas quantidades de PA6 e das blendas poliméricas foram tomadas de diferentes partes da amostra e foram determinados teores de carbono, hidrogênio e nitrogênio, utilizando um equipamento da Perkin Elmer, model 2400 CHN. Este procedimento foi utilizado para se avaliar, a partir da percentagem de nitrogênio, a quantidade de PA6 na amostra, e estimar a homogeneidade da amostra por meio de reprodutibilidade dos resultados obtidos, com as diferentes amostras.

Foram analisadas as micrografias obtidas por microscopia eletrônica de varredura (Philips - modelo XL 30) das superfícies de fratura das amostras fraturadas em nitrogênio líquido, assim como das superfícies de fratura obtidas pelo teste de impacto. Uma fina camada de ouro foi depositada sobre as amostras, utilizando-se um metalizador da International Scientific Instruments, modelo P-S2. Análises das imagens foram realizadas com o auxílio do software AnalySis Pró, versão 2.11 .

Os ensaios de impacto Izod foram realizados de acordo com a norma ASTM D 256-54T, em uma máquina de impacto da Custom Scientific Instruments, à temperatura ambiente. Os corpos de prova da PA6 e das blendas poliméricas, inicialmente foram obtidos por prensagem e posteriormente foram cortados segundo o molde recomendado pela norma. Foram realizados dez ensaios sem entalhe para cada composição estudada da blenda.

Os ensaios de tensão/deformação foram realizados em um equipamento Instron ${ }^{\mathrm{TM}}$, modelo 8501, com um sistema de 
teste mecânico servo-hidráulico, equipado com garras pneumáticas. A taxa de estiramento foi de $5 \mathrm{~cm}$ por minuto e as amostras foram preparadas segundo a norma ASTM D638M.

\section{Resultados e Discussão}

Entre as diversas técnicas para avaliação da miscibilidade de componentes nas blendas poliméricas, tem sido utilizada a verificação de deslocamentos das bandas de absorção no infravermelho em relação ao número de onda, devido a interações entre grupos funcionais. Pode-se citar como exemplo, a observação de mudanças no número de onda da absorção devido ao estiramento do grupo hidroxila em blendas de poli(vinil fenol)/poli(vinil metil cetona) ${ }^{[13]}$, em interações específicas entre blendas de poli(estireno-co-ácido metacrílico) com copolímeros contendo grupos vinilpirrolidona e vinilpiridina ${ }^{[14]}$ ou blendas contendo metal as quais formam complexos de coordenação ${ }^{[15]}$. Nos estudos do efeito da adição de poli(oxipropileno diamina) em blendas de polipropileno/elastômeros enxertados com anidrido maleico, Phan e colaboradores ${ }^{[16]}$ consideraram o aparecimento de duas bandas em $1703 \mathrm{e} 1774 \mathrm{~cm}^{-1}$ devido a vibração de estiramento da carbonila do grupo imídico, formado durante o processo de mistura entre o anidrido maleico e a amina presentes no agente compatibilizante.

Na faixa de 2000 a $1000 \mathrm{~cm}^{-1}$ as bandas de absorção de estiramento da carbonila presente no anidrido maleico e no ácido maleico ocorrem a $1780-1720 \mathrm{~cm}^{-1} \mathrm{e} 1707 \mathrm{~cm}^{-1}$, respectivamente ${ }^{[13]}$. Os espectros de infravermelho do EPDMg-AM puro e da blenda PA6/EPDM-g-AM na composição de $80 / 20$ m/m são apresentados na Figura 1, com inserção da deconvolução das bandas na faixa de 1800 a $1680 \mathrm{~cm}^{-1}$. A banda devido a vibração do grupo carbonila presente na cadeia polimérica da PA6 é intensa e ocorre a $1640 \mathrm{~cm}^{-1}$, enquanto que as bandas de absorção devido ao anidrido maleico são de menor intensidade e ocorrem em maiores números de onda ${ }^{[13]}$. A ocorrência de absorção devido a carbonila de ácido maleico no espectro do EPDM-g-AM puro deve-se a facilidade de conversão de anidrido maleico à ácido maleico, nas condições de processamento das blendas. $\mathrm{O}$ aparecimento de bandas em 1703 e $1764 \mathrm{~cm}^{-1}$ no espectro infravermelho da blenda PA6/EPDM-g-AM é devido a absorção da carbonila do grupo imida formado na interface dos domínios de elastômero e da matriz de PA6, sendo resultante da reação do grupo amino da PA6 e o anidrido maleico do agente compatibilizante ${ }^{[17]}$. A permanência da banda em $1780 \mathrm{~cm}^{-1}$ sugere a presença de anidrido maleico na blenda, indicando que parte do anidrido maleico adicionado à mistura não reagiu. Resultados análogos foram observados para o sistema PA6/EPM-g-AM.

Os valores da temperatura de fusão $\left(\mathrm{T}_{\mathrm{f}}\right)$, de transição vítrea $\left(T_{g}\right)$ e a variação de entalpia de fusão $\left(\Delta H_{f}\right)$ dos componentes puros e das blendas foram calculados a partir da segunda corrida de aquecimento, conforme descrito na parte experimental. A variação de entalpia de fusão do componente semicristalino foi corrigida para um grama de PA6 a partir
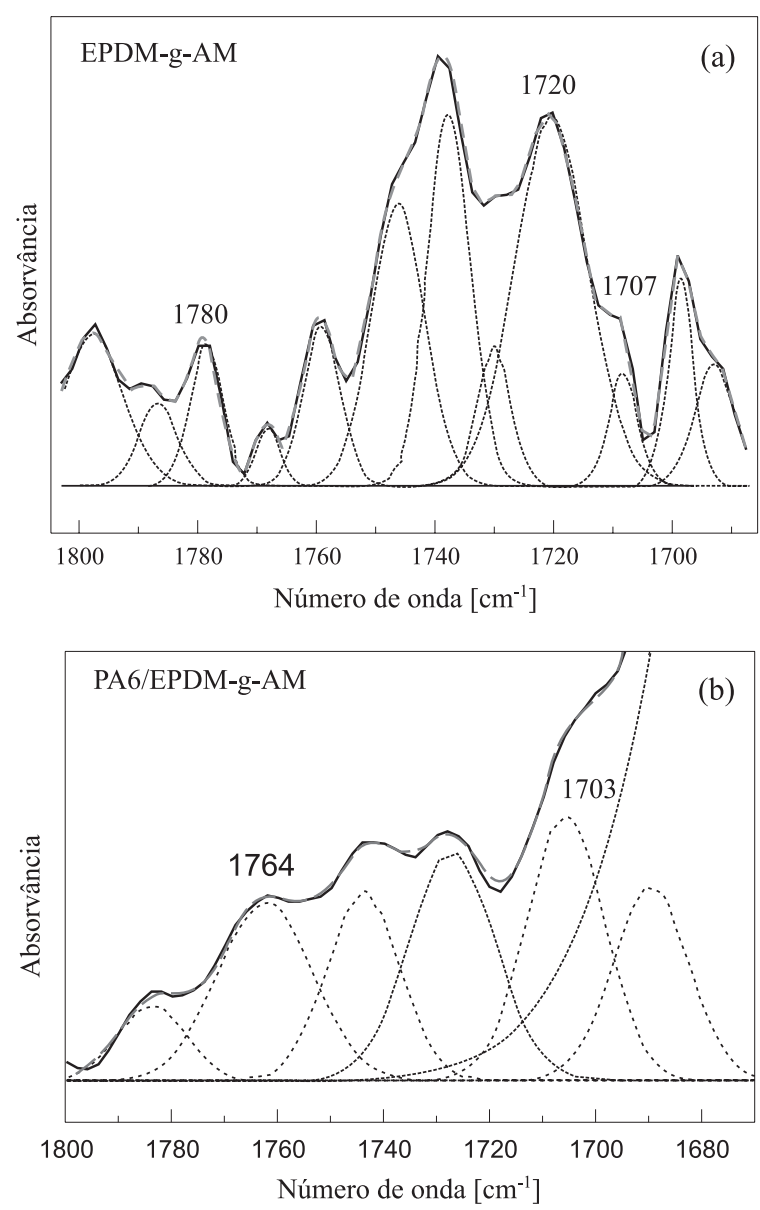

Figura 1. Espectro de infravermelho com transformada de Fourier na região de 1680 a $1800 \mathrm{~cm}^{-1}$ de (a) EPDM-g-MA puro e (b) blenda de PA6/ EPDM-g-AM na composição de 80/20 m/m.

da quantidade deste componente na blenda. Os valores experimentais de $\mathrm{T}_{\mathrm{g}}, \mathrm{T}_{\mathrm{c}}, \mathrm{T}_{\mathrm{m}}$ e $\Delta \mathrm{H}_{\mathrm{m}}$ para os componentes puros e para as blendas, apresentados na Tabela 1 , são da mesma ordem de grandeza sugerindo que a presença do componente amorfo não altera a temperatura de fusão e a variação de entalpia de fusão é proporcional a percentagem de componente cristalino na blenda. A não observação de um único valor de $\mathrm{T}_{\mathrm{g}}$, intermediária aos componentes puros, sugere a imiscibilidade do sistema, os quais concordam com os resultados de espectroscopia de infravermelho.

Conforme discutido anteriormente, a adição de EPDM-g$\mathrm{AM}$ ou EPM-g-AM à mistura binária altera quimicamente a interação entre os componentes na interface das fases da mistura imiscível. Este efeito pode levar a uma redução do tamanho dos domínios e maior homogeneidade destes na matriz. Para uma avaliação experimental desta dispersão, foi utilizado a técnica de análise elementar de CHN. A Tabela 2 apresenta a percentagem de nitrogênio presente na PA6 pura, na blenda binária de PA6/EPDM-g-AM $(80 / 20 \mathrm{~m} / \mathrm{m})$ e em presença de EPDM enxertado com anidrido maleico, mantendo a mesma percentagem de PA6. A presença de agente compatibilizante aumenta a homogeneidade da mistura em relação as blendas não compatibilizadas. A redução no tamanho das partículas da fase elastomérica com a adição de EPDM 
Tabela 1. Temperaturas de transição para os componentes puros e blendas e variação de entalpia de fusão para o componente semicristalino

\begin{tabular}{ccccc}
\hline $\begin{array}{c}\text { PA6/EPDM/EPDM-g-AM/EPM-g-AM } \\
\mathbf{m} / \mathbf{m} / \mathbf{m} / \mathbf{m}\end{array}$ & $\begin{array}{c}\mathbf{T}_{\mathrm{g}} \\
\left({ }^{\circ} \mathbf{C}\right)\end{array}$ & $\begin{array}{c}\mathbf{T}_{\mathbf{c}} \\
\left({ }^{\circ} \mathbf{C}\right)\end{array}$ & $\begin{array}{c}\mathbf{T}_{\mathrm{f}} \\
\left({ }^{\circ} \mathbf{C}\right)\end{array}$ & $\begin{array}{c}\Delta \mathbf{H}_{\mathrm{f}} \\
(\mathbf{c a l} / \mathbf{g})\end{array}$ \\
\hline $00 / 100 / 00 / 00$ & -50 & - & - & - \\
$00 / 00 / 100 / 00$ & -48 & - & - & - \\
$00 / 00 / 00 / 100$ & -45 & - & - & 23 \\
$100 / 00 / 00 / 00$ & 41 & 68 & 224 & 18 \\
$80 / 20 / 00 / 00$ & 43 & 68 & 225 & 18 \\
$80 / 15 / 05 / 00$ & 43 & 68 & 225 & 18 \\
$80 / 10 / 10 / 00$ & 42 & 69 & 225 & 18 \\
$80 / 00 / 20 / 00$ & 43 & 69 & 225 & 19 \\
$80 / 15 / 00 / 05$ & 42 & 68 & 225 & 18 \\
$80 / 10 / 00 / 10$ & 41 & 67 & 224 & 18 \\
$80 / 00 / 00 / 20$ & 43 & 69 & 225 & \\
\hline
\end{tabular}

funcionalizado pode ser relacionado com a estabilização da morfologia da blenda e redução da tensão interfacial entre os componentes.

A Figura 2 mostra as micrografias das fraturas de filmes das blendas de PA6/EPDM/EPDM-g-AM na percentagem em massa de $80 / 15 / 5$, antes e após a extração da fase dispersa com clorofórmio, durante $24 \mathrm{~h}$. Os vazios observados na micrografia da Figura 2 (b) correspondem ao espaços ocupados pelo elastômero, antes do processo de solubilização. Micrografias de fraturas dos filmes das blendas PA6/EPDM na composição de $80 / 20 \mathrm{~m} / \mathrm{m}$ e na presença de agente compatibilizante na percentagem de 5, 10 e $20 \%$ mantendo- se constante a percentagem de PA6 em $80 \%$, tratadas com solvente são apresentadas na Figura 3. O aumento da quantidade de agente compatibilizante na mistura induz a uma redução no tamanho da fase dispersa, sendo que a presença de apenas 5\% de EPDM enxertado com anidrido maleico altera significativamente a morfologia da blenda.

A resistência ao impacto das amostras do PA6 puro e das blendas são apresentados na Tabela 3. Os resultados experimentais apresentam desvio padrão relativamente alto, provavelmente devido a sensibilidade da medida em relação a absorção de umidade e variações do grau de cristalização. A redução na resistência ao impacto Izod para a composição

Tabela 2. Percentagem de nitrogênio em diferentes amostras, determinadas por análise elementar CHN

\begin{tabular}{lcccc}
\hline Blenda & Amostra & $\begin{array}{c}\text { Percentagem } \\
\text { nitrogênio }\end{array}$ & $\begin{array}{c}\text { Composição de PA6 } \\
\text { na blenda }\end{array}$ & Desvio padrão \\
\hline PA6 & 1 & 12,35 & 100,0 & \\
& 2 & 12,45 & 100,0 & 0,04 \\
& 3 & 12,35 & 100,0 & \\
\hline PA6/EPDM & 1 & 11,04 & 89,2 & 4,9 \\
& 2 & 9,30 & 75,1 & \\
\hline PA6/EPDM/EPDM-g-MA & 1 & 10,31 & 83,2 & 0,6 \\
& 3 & 9,57 & 77,3 & \\
\hline & 2 & 9,73 & 78,6 & \\
& 3 & 9,55 & 77,2 & \\
& & & & \\
& & &
\end{tabular}



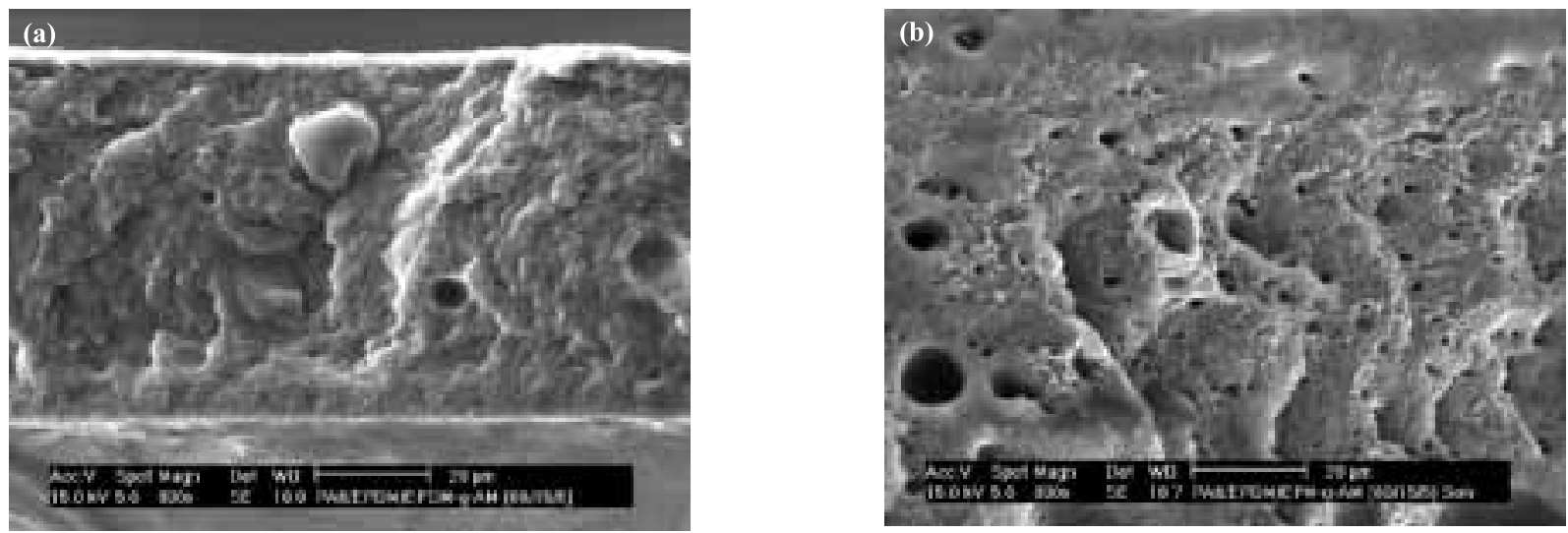

Figura 2. Micrografias obtidas em microscópio eletrônico de varredura de fraturas de filmes da blenda de PA6/EPDM/EPDM-g-MA na composição de 80/ $15 / 5 \mathrm{~m} / \mathrm{m} / \mathrm{m}$ (a) sem tratamento e (b) com tratamento em clorofórmio durante $24 \mathrm{~h}$.

binária PA6/EPDM em relação à blenda PA6/EPDM/EPDMg-AM deve-se provavelmente a fraca adesão entre as fases. Os diâmetros dos domínios em blendas binárias com pequenas quantidades de EPDM-g-AM com um grau de enxerto de anidrido maleico de $1,7 \%$, são da ordem de 0,03 a 0,60 $\mu \mathrm{m}$, faixa considerada ideal para obtenção de materiais tenazes $^{[18]}$. Entretanto, valores de resistência ao impacto Izod superiores ao valor correspondente a PA6 foram obtidos para as blendas PA6/EPDM-g-AM. A maior quantidade de anidrido maleico favorece a formação do copolímero na interface, diminuindo a tensão interfacial e aumentando a
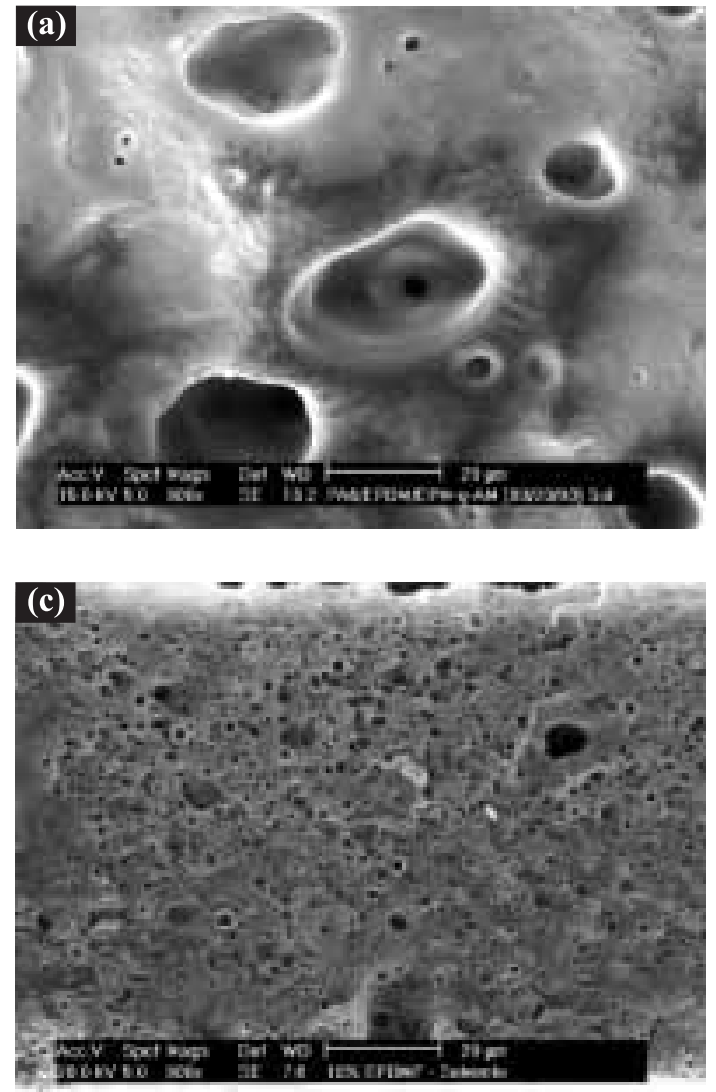

adesão entre os domínios e a matriz na blenda polimérica.

$\mathrm{Na}$ Figura 4 tem-se as micrografias de amostras da blenda binária na composição de $80 / 20 \mathrm{~m} / \mathrm{m}$ e com agente compatibilizante fraturadas mecanicamente sob impacto Izod. A morfologia das blendas são típicas de amostras tenazes, com fratura dúctil, sendo que devido a fraca interação interfacial existem vazios entre os domínios de borracha e da matriz, os quais diminuem com o aumento da percentagem de agente compatibilizante na amostra (Figura 4). As blendas com $20 \%$ de elastômero enxertado com anidrido maleico apresentaram uma redução dos domínios arranca-
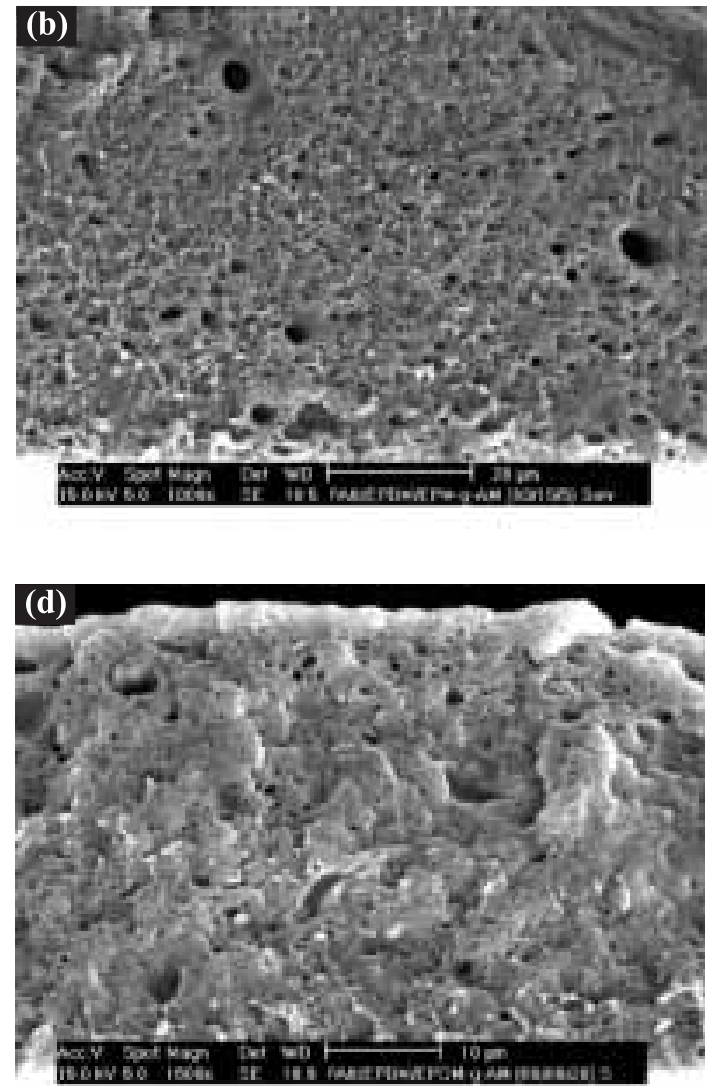

Figura 3. Micrografia obtida em microscópio eletrônico de varredura de fraturas de filmes de blendas de PA6/EPDM/EPDM-g-MA na composição de (a) $80 / 20 / 00$, (b) $80 / 15 / 5$, (c) $80 / 10 / 10$ e (d) $80 / 00 / 20 \mathrm{~m} / \mathrm{m} / \mathrm{m}$. 
Tabela 3. Resistência ao impacto Izod das blendas de PA6/EPDM/EPDMg-MA à temperatura ambiente

\begin{tabular}{ccc}
\hline PA6/EPDM/EPDM-g-MA & $\begin{array}{c}\text { Resistência ao } \\
\text { Impacto Izod } \\
(\mathbf{J} / \mathbf{m})\end{array}$ & $\begin{array}{c}\text { Desvio } \\
\text { Padrão }\end{array}$ \\
\hline $100 / 00 / 00$ & 136 & 8,0 \\
$80 / 20 / 00$ & 45 & 6,5 \\
$80 / 15 / 05$ & 51 & 4,3 \\
$80 / 10 / 10$ & 68 & 4,4 \\
$80 / 00 / 20$ & 167 & 4,0 \\
\hline
\end{tabular}

dos no processo de fratura, devido provavelmente a maior quantidade de copolímero formado na interface matriz/fase dispersa. (Figura $4 \mathrm{~d}$ e f). A presença destes vazios aumenta a concentração de tensões locais, as quais podem acarretar em uma falha prematura da blenda.

A curva de tensão/deformação para a blenda PA6/EPDM mostra características de material duro e quebradiço (Figura 5). O aumento da deformação plástica para as blendas compatibilizadas é devido ao aumento da capacidade de absorção de energia. A quantidade de compatibilizante nos sistemas modifica significativamente a morfologia das misturas, com redução do tamanho dos domínios, maior homogeneidade na dispersão do elastômero e adesão interfacial entre as fases.
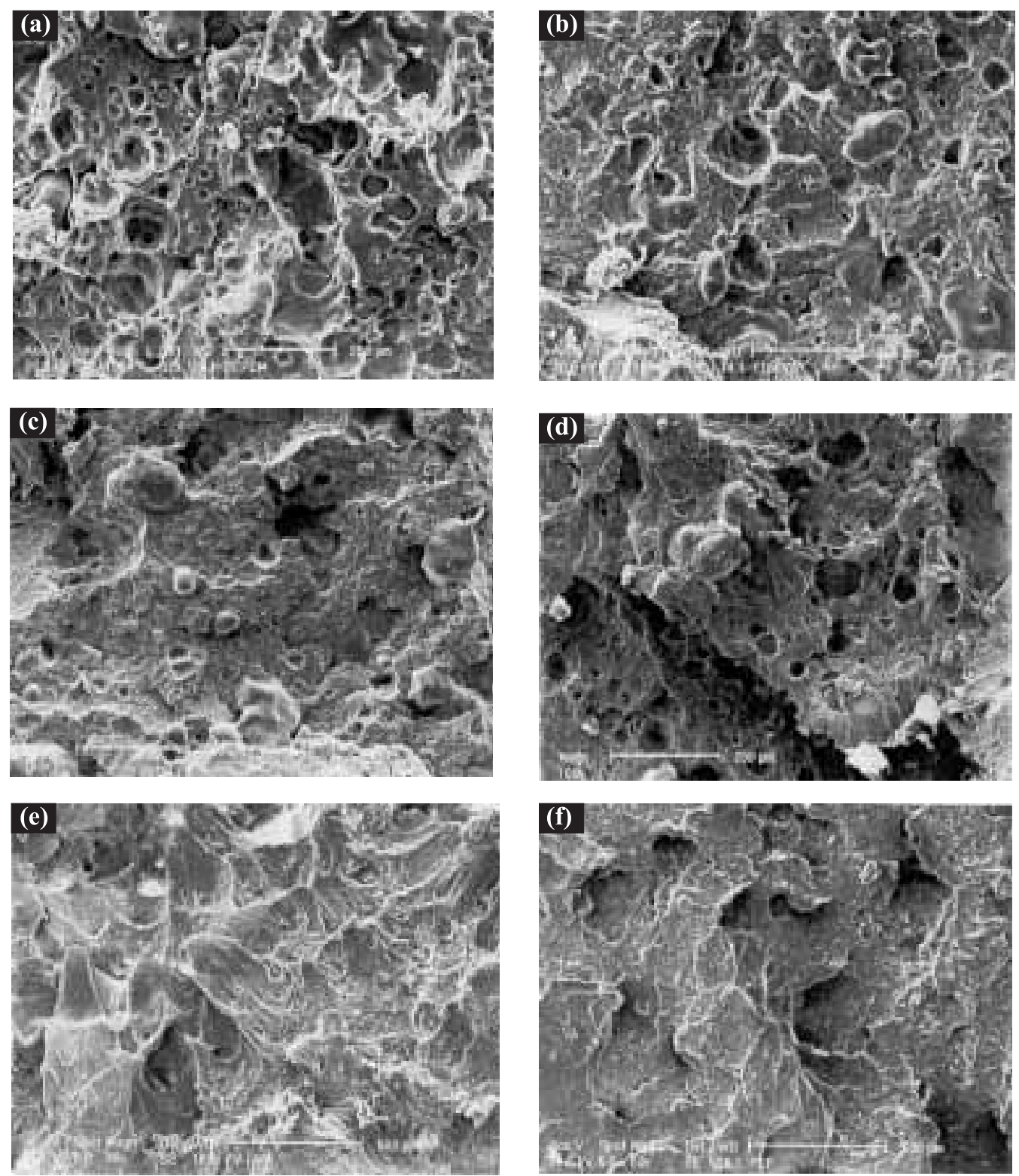

Figura 4. Micrografias das superfícies de fratura dos corpos de prova após os experimentos de força de impacto Izod, com ampliação de 100 vezes, das blendas: (a) PA6/EPDM (80/20), (b) PA6/EPDM/EPDM-g-MA (80/15/5), (c) PA6/EPDM/EPDM-g-MA (80/10/10), (d) PA6/EPDM/EPM-g-MA (80/10/10), (e) PA6/EPM-g-MA (80/20) e (f) PA6/EPDM-g-MA (80/20) m/m. 


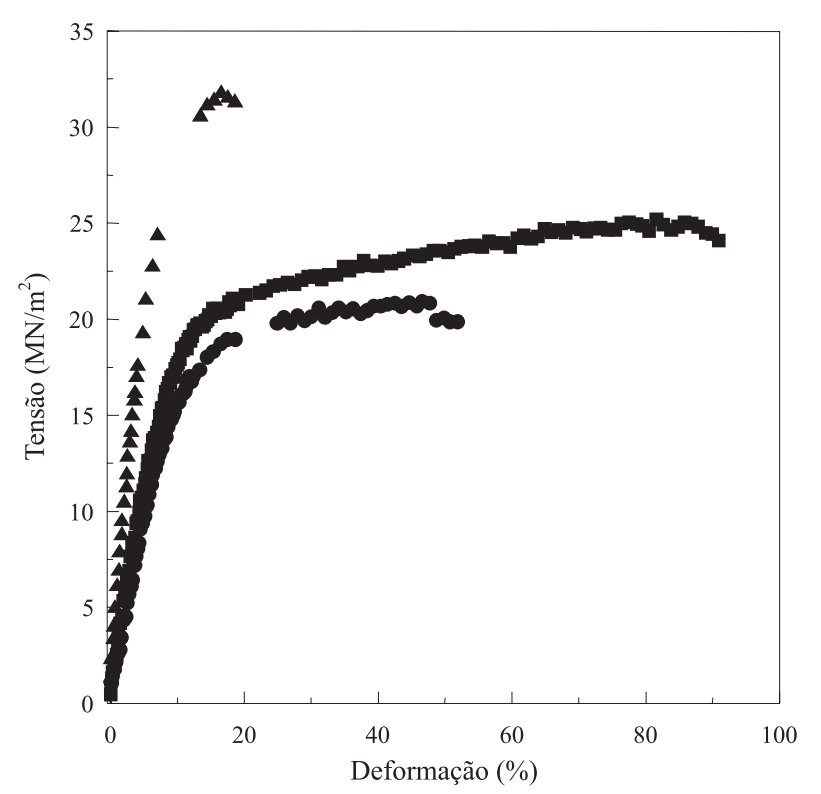

Figura 5. Curvas da tensão aplicada em função da deformação de corpos de prova das blendas : (•) PA6/EPDM (80/20), (•) PA6/EPM-g-MA (80/ 20) e (•) PA6/EPDM/EPDM-g-MA (80/10/10).

\section{Conclusão}

A adição de elastômero enxertado com anidrido maleico à blendas binárias imiscíveis de PA6/EPDM aumenta a homogeneidade da dispersão dos domínios de EPDM na matriz de PA6, com a redução do tamanho dos domínios e formação de um copolímero na interface. Este copolímero resultante da reação do grupo amino da PA6 com o anidrido maleico do agente compatibilizante, induz mudanças na morfologia das blendas e na adesão interfacial matriz/domínios as quais influenciam nas propriedades mecânicas, ou seja, na resistência ao impacto Izod e no comportamento tensão/ deformação.

\section{Referências Bibliográficas}

1. Newman, S. - Rubber modification of plastics, In: Paul, D. R. and Newman, S. Polymer Blend, New York, Academic Press, v.2., 63 (1978).

2. Maglio, G; Palumbo, R. - The role of interfacial agents in polymer blends. Polymer Blends, Processing,
Morphology and Properties. New York, Plenum Press, v.2, 41 (1984).

3. Ahn, T. O.; Hong, S. C.; Jeong, H. M.; Kim, J. H. - Polymer, 38, 207 (1997).

4. Horiuchi, S.; Matchariyakul, N.; Yase, K.; Kitano, T.; Choi, H. K.; Lee, Y. M. - Polymer, 38, 59 (1997).

5. Thomas, S.; Groeninckx, G. - Journal Applied Polymer Science, 71, 1405 (1999).

6. Thomas, S.; Groenincks, G. - Polymer, 40, 5799 (1999).

7. Roeder, J.; Oliveira, R. V. B.; Gonçalves, M. C.; Soldi, V.; Pires, A. T. N. - Polymer Testing, 21, 815 (2002).

8. Becker, D.; Roeder, J.; Oliveira, R. V. B.; Soldi, V.; Pires, A. T. N. - Polymer Testing, 22, 225 (2003).

9. Pagnoulle, C.; Jérôme, R. - Polymer, 42, 1893 (2001).

10. Inoue, T.; Suzuki, T. - Journal of Applied Polymer Science, 59 (1996) 1443. Ahn, T. O.; Hong S. C.; Jeong, H. M.; Kim, J. H. - Polymer, 38, 207 (1997).

11. Shing-Chung W.; Yiu-Wing M. - Polymer, 41, 5471 (2000).

12. Lu, M.; Keskkula, H.; Paul, D. R. - Journal of Applied Polymer Science, 58, 1175 (1995).

13. Barra, G. M. O.; Crespo, J. S.; Bertolino, J. R.; Soldi, V.; Pires, A. T. N. - Journal of the Brazilian Chemical Society, 10, 31 (1999).

14. Coleman, M. M.; Painter, P.C. - Applied Spectroscopy Review, 20, 255 (1984).

15. Painter, P. C.; Coleman M. M. - Macromolecules, 34, 8390 (2001).

16. Qin, C.; Pires, A. T. N.; Belfiore, L. A. - Macromolecules, 24, 666 (1991).

17. Phan, T. T. M.; Denicola, A. J. Jr.; Schadler, L. S. - Journal of Applied Polymer Science, 68, 1452 (1998).

18. Lawson, D. F.; Hergenkother, W. L.; Matlock, M. G. Journal of Applied Polymer Science, 39, 2331 (1990).

Recebido: $15 / 04 / 02$

Aprovado: 18/12/02 